Supplement of Biogeosciences Discuss., 11, 8969-9011, 2014

http://www.biogeosciences-discuss.net/bgd-11-8969-2014/

doi:10.5194/bgd-11-8969-2014-supplement

(C) Author(s) 2014. CC Attribution 3.0 License.

(c) (i)

Supplement of

\title{
Contrasting photosynthetic characteristics of forest vs. savanna species (far North Queensland, Australia)
}

\section{K. J. Bloomfield et al.}

Correspondence to: K. J. Bloomfield (keith.bloomfield@anu.edu.au) 
Table S1. List of species measured with Site code (Table 1) and $\Phi$ (Table S2) for the tropical moist forest species.

\begin{tabular}{|c|c|c|c|c|}
\hline Site & Type & Species & $\Phi$ (forest species) & $n$ \\
\hline KBL-01 & Forest & Alangium villosum & Sub-canopy & 4 \\
\hline KBL-01 & Forest & Aleurites rocking hamensis & Tall pioneer & 4 \\
\hline KBL-03 & Forest & Apodytes brachystylis & Sub-canopy & 4 \\
\hline KCR-01 & Forest & Ardisia brevipedata & Sub-canopy & 4 \\
\hline CTC-01 & Forest & Argyrodendron peralatum & Upper canopy & 4 \\
\hline KBL-03 & Forest & Beilschmiedia tooram & Upper canopy & 1 \\
\hline CTC-01 & Forest & Cardwellia sublimis & Tall pioneer & 4 \\
\hline KCR-01 & Forest & Cardwellia sublimis & Tall pioneer & 5 \\
\hline KBL-01 & Forest & Castanospora alphandii & Upper canopy & 1 \\
\hline CTC-01 & Forest & Cleistanthus myrianthus & Upper canopy & 3 \\
\hline DCR-02 & Savanna & Corymbia citriodora & & 3 \\
\hline DCR-02 & Savanna & Corymbia clarksoniana & & 3 \\
\hline DCR-01 & Savanna & Corymbia intermedia & & 1 \\
\hline KBL-02 & Savanna & Corymbia intermedia & & 4 \\
\hline KBL-01 & Forest & Elaeocarpus grandis & Tall pioneer & 1 \\
\hline KBL-03 & Forest & Elaeocarpus largiflorens & Upper canopy & 4 \\
\hline DCR-02 & Savanna & Eucalyptus portuensis & & 4 \\
\hline KCR-01 & Forest & Eupomatia laurina & Sub-canopy & 5 \\
\hline CTC-01 & Forest & Haplostichanthus ramiflorus & Sub-canopy & 4 \\
\hline KBL-03 & Forest & Litsea leefeana & Tall pioneer & 1 \\
\hline DCR-01 & Savanna & Lophostemon suaveolens & & 3 \\
\hline KBL-02 & Savanna & Lophostemon suaveolens & & 4 \\
\hline CTC-01 & Forest & Mallotus mollissimus & Small pioneer & 4 \\
\hline KBL-01 & Forest & Neisosperma poweri & Sub-canopy & 4 \\
\hline KBL-01 & Forest & Neolitsea dealbata & Small pioneer & 4 \\
\hline KBL-03 & Forest & Neolitsea dealbata & Small pioneer & 4 \\
\hline KCR-01 & Forest & Neolitsea dealbata & Small pioneer & 6 \\
\hline DCR-01 & Savanna & Planchonia careya & & 6 \\
\hline KCR-01 & Forest & Polyscias elegans & Tall pioneer & 4 \\
\hline CTC-01 & Forest & Pseuduvaria froggattii & Sub-canopy & 4 \\
\hline KBL-03 & Forest & Sloanea australis & Upper canopy & 4 \\
\hline KBL-03 & Forest & Symplocos hayesii & Sub-canopy & 4 \\
\hline KBL-03 & Forest & Synima cordierorum & Sub-canopy & 1 \\
\hline KCR-01 & Forest & Toechima erythrocarpum & Sub-canopy & 4 \\
\hline \multirow[t]{3}{*}{ KBL-03 } & Forest & Wilkiea angustifolia & Sub-canopy & 5 \\
\hline & & \multicolumn{2}{|l|}{ Total number of $A \leftrightarrow C_{i}$ curves } & 125 \\
\hline & & \multicolumn{2}{|c|}{ Number of Site + Species combinations with replicates $\geq 3$} & 28 \\
\hline
\end{tabular}


Table S2. Plant functional type $(\Phi)$ assigned to the forest species. The classification assigned to each species is based on light requirement and adult stature.

\begin{tabular}{ll}
\hline Plant functional type $(\Phi)$ & Description \\
\hline Small pioneer & Small stature pioneer, light demanding \\
Sub-canopy & Sub canopy, shade tolerant \\
Tall pioneer & Tall pioneer, light demanding \\
Upper-canopy & Upper canopy, shade tolerant, but well adapted to sunlit conditions \\
\hline
\end{tabular}


Table S3. Results of standardised major axis (SMA) bivariate relationships testing the null hypothesis that there was no difference between Forest and Savanna trees in slope (LR, Likelihood ratio statistic), intercept or axis shift (Wald statistic). For each bivariate relationship the assumption of linearity, for the dataset as a whole, was tested using Pearson's correlation (r). The dataset here excludes the four outlying points identified on Figure 2a.

\begin{tabular}{|c|c|c|c|c|c|c|c|c|c|c|}
\hline \multirow[b]{3}{*}{ Response } & \multirow[b]{3}{*}{ Bivariate } & \multicolumn{3}{|c|}{ Test for linear relationship } & \multicolumn{6}{|c|}{ Significance of difference between Vegetation classes in } \\
\hline & & & & & Slo & & Eleva & & Shi & \\
\hline & & df & $\mathrm{r}$ & $p$ & $\mathrm{LR}$ & $p$ & Wald & $p$ & Wald & $p$ \\
\hline$[\mathrm{P}]_{\mathrm{a}} \leftrightarrow$ & {$[\mathrm{N}]_{\mathrm{a}}$} & 103 & 0.89 & $<0.0001$ & 7.58 & 0.0059 & NA & NA & NA & NA \\
\hline$[\mathrm{P}]_{\mathrm{m}} \leftrightarrow$ & {$[\mathrm{N}]_{\mathrm{m}}$} & 103 & 0.90 & $<0.0001$ & 3.41 & 0.0646 & 3.74 & 0.053 & 19.10 & $<0.0001$ \\
\hline$[\mathrm{N}]_{a} \leftrightarrow$ & $M_{\mathrm{a}}$ & 103 & 0.63 & $<0.0001$ & 13.83 & 0.0002 & NA & NA & NA & NA \\
\hline$[\mathrm{P}]_{\mathrm{a}} \leftrightarrow$ & $M_{\mathrm{a}}$ & 103 & 0.53 & $<0.0001$ & 3.08 & 0.0794 & 10.00 & 0.002 & 45.74 & $<0.0001$ \\
\hline$A_{\max , \mathrm{a}} \leftrightarrow$ & {$[N]_{a}$} & 103 & 0.67 & $<0.0001$ & 16.09 & $<0.0001$ & NA & NA & NA & NA \\
\hline$A_{\max , \mathrm{a}} \leftrightarrow$ & {$[P]_{a}$} & 103 & 0.63 & $<0.0001$ & 3.98 & 0.0459 & NA & NA & NA & NA \\
\hline$A_{\max , \mathrm{a}} \leftrightarrow$ & $M_{\mathrm{a}}$ & 103 & 0.55 & $<0.0001$ & 0.02 & 0.8805 & 0.19 & 0.659 & 63.41 & $<0.0001$ \\
\hline$A_{\text {max }, \mathrm{m}} \leftrightarrow$ & {$[\mathrm{N}]_{\mathrm{m}}$} & 103 & 0.69 & $<0.0001$ & 0.77 & 0.3809 & 40.36 & $<0.0001$ & 4.33 & 0.037 \\
\hline$A_{\max , \mathrm{m}} \leftrightarrow$ & {$[P]_{m}$} & 103 & 0.70 & $<0.0001$ & 0.02 & 0.8976 & 26.28 & $<0.0001$ & 2.40 & 0.121 \\
\hline$A_{\max , \mathrm{m}} \leftrightarrow$ & $M_{\mathrm{a}}$ & 107 & -0.23 & 0.017 & 7.53 & 0.0061 & NA & NA & NA & NA \\
\hline$V_{\text {cmax }, \mathrm{a}} \leftrightarrow$ & {$[N]_{a}$} & 103 & 0.65 & $<0.0001$ & 15.42 & $<0.0001$ & NA & NA & NA & NA \\
\hline$V_{c \max , \mathrm{m}} \leftrightarrow$ & {$[\mathrm{N}]_{\mathrm{m}}$} & 103 & 0.68 & $<0.0001$ & 0.09 & 0.7686 & 63.93 & $<0.0001$ & 2.59 & 0.107 \\
\hline$V_{\text {cmax }, \mathrm{a}} \leftrightarrow$ & {$[P]_{a}$} & 103 & 0.62 & $<0.0001$ & 3.51 & 0.0611 & 19.67 & $<0.0001$ & 41.12 & $<0.0001$ \\
\hline$V_{\text {cmax }, \mathrm{m}} \leftrightarrow$ & {$[P]_{m}$} & 103 & 0.70 & $<0.0001$ & 0.56 & 0.4549 & 44.50 & $<0.0001$ & 1.17 & 0.279 \\
\hline$J_{\max , a} \leftrightarrow$ & {$[N]_{a}$} & 103 & 0.69 & $<0.0001$ & 13.72 & 0.0002 & NA & NA & NA & NA \\
\hline$J_{\max , m} \leftrightarrow$ & {$[\mathrm{N}]_{\mathrm{m}}$} & 103 & 0.70 & $<0.0001$ & 0.45 & 0.5034 & 49.83 & $<0.0001$ & 3.86 & 0.050 \\
\hline$J_{\max , a} \leftrightarrow$ & {$[P]_{a}$} & 103 & 0.66 & $<0.0001$ & 2.85 & 0.0917 & 13.99 & 0.0002 & 39.73 & $<0.0001$ \\
\hline$J_{\max , \mathrm{m}} \leftrightarrow$ & {$[\mathrm{P}]_{\mathrm{m}}$} & 103 & 0.72 & $<0.0001$ & 0.14 & 0.7086 & 32.03 & $<0.0001$ & 2.07 & 0.150 \\
\hline DM: $\mathrm{H}_{2} \mathrm{O} \leftrightarrow$ & $M_{\mathrm{a}}$ & 107 & 0.62 & $<0.0001$ & 7.84 & 0.0051 & NA & NA & NA & NA \\
\hline$d \leftrightarrow$ & $M_{\mathrm{a}}$ & 107 & 0.24 & 0.0113 & 21.52 & $<0.0001$ & NA & NA & NA & NA \\
\hline$\rho \leftrightarrow$ & $M_{\mathrm{a}}$ & 104 & 0.82 & $<0.0001$ & 5.58 & 0.0181 & NA & NA & NA & NA \\
\hline$A_{\mathrm{N}} \leftrightarrow$ & $M_{\mathrm{a}}$ & 103 & 0.34 & 0.0004 & 0.24 & 0.6249 & 0.86 & 0.353 & 58.44 & $<0.0001$ \\
\hline
\end{tabular}


Figure S1. Pair-wise plot of response and explanatory variables. The upper panel contains estimated pairwise correlations and the font size is proportional to the absolute value of the estimated correlation coefficient. Significance codes: $* * *<0.001, * *<0.01, *<0.05, .<0.01$. The diagonal panel contains histograms and the lower panel scatterplots with a local regression smoother added to aid visual interpretation. The selected variables are photosynthetic capacity on an area basis as response with six continuous explanatory variables (spanning leaf morphology and nutrient levels). Trait units are $A_{\max , a}$ ( $\mu \mathrm{mol} \mathrm{CO} \mathrm{CO}_{2}^{-2} \mathrm{~s}^{-1}$ ), Maximum conductance $\left(\mathrm{mol} \mathrm{m}^{-2} \mathrm{~s}^{-1}\right)$, Leaf mass per unit area $\left(\mathrm{g} \mathrm{m}^{-2}\right.$ ), Leaf dry matter content $\left(\mathrm{g} \mathrm{g}^{-1}\right)$, Leaf thickness $(\mu \mathrm{m})$, Total leaf nitrogen per unit area $\left(\mathrm{g} \mathrm{m}^{-2}\right)$, Total leaf phosphorus per unit area $\left(\mathrm{mg} \mathrm{m}^{-2}\right)$.

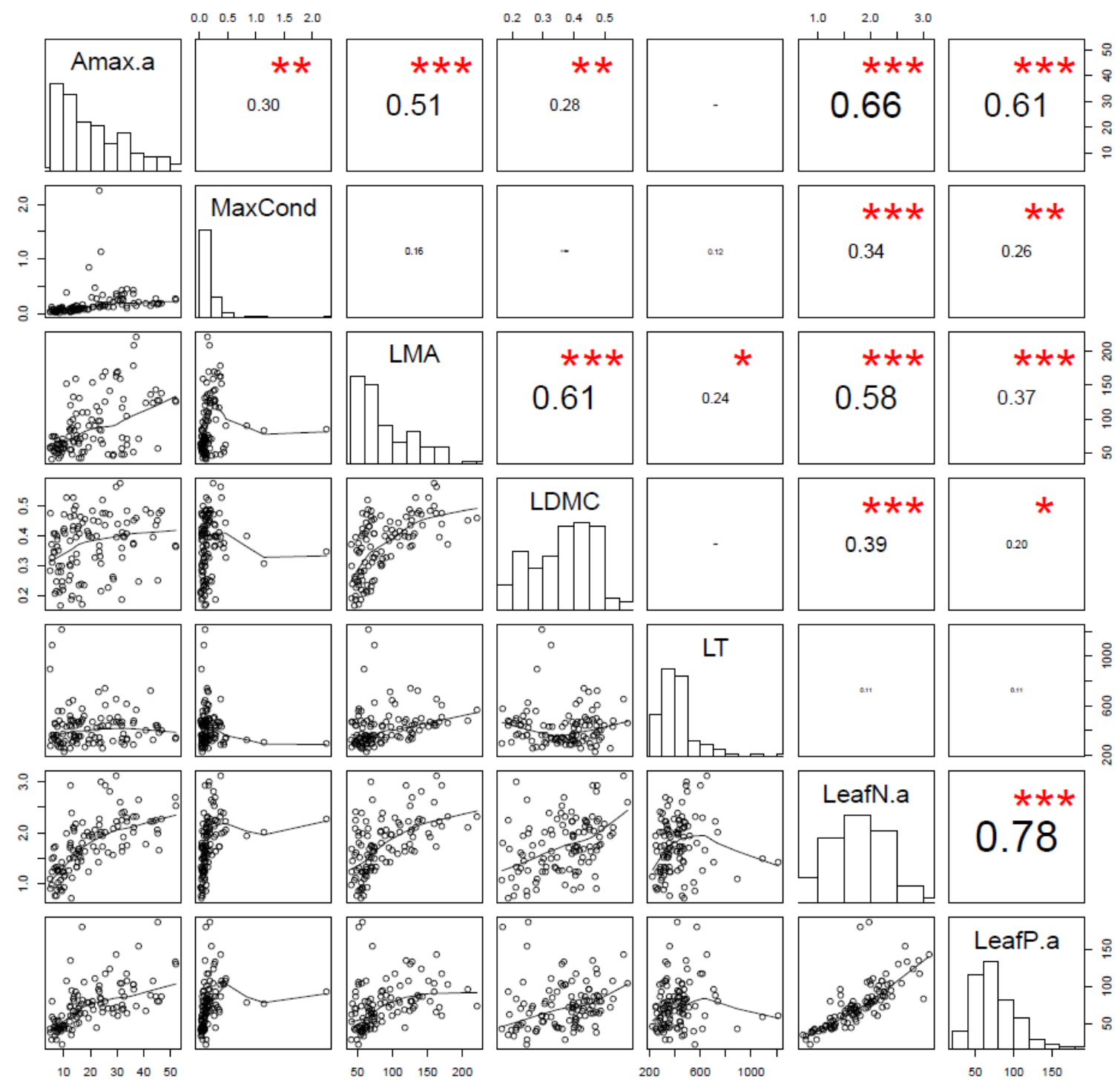


Figure S2: Model validation graphs for the random intercept mixed effects model (Eq. 2). Standardised residuals are plotted against fitted values and each of the explanatory factors and variables used in the model's fixed component.

(a)

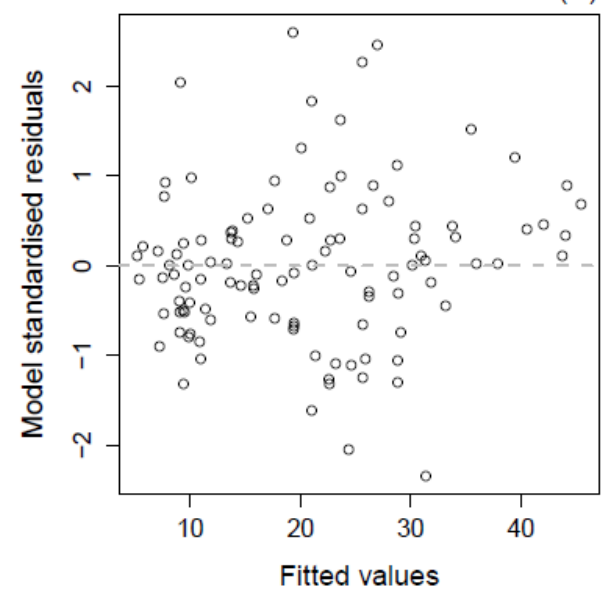

(c)

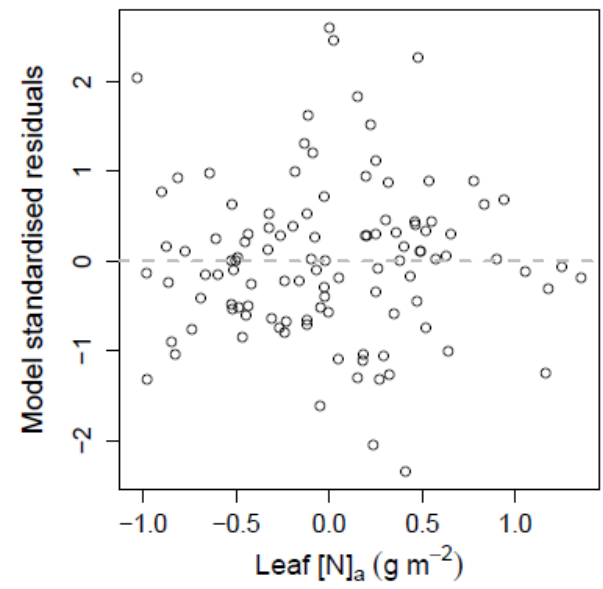

(b)

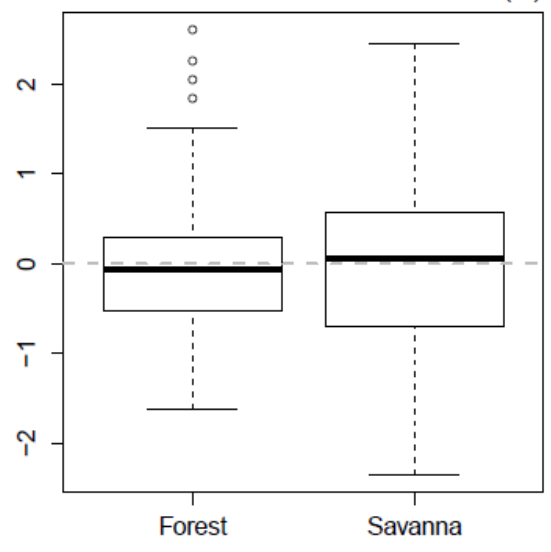

(d)

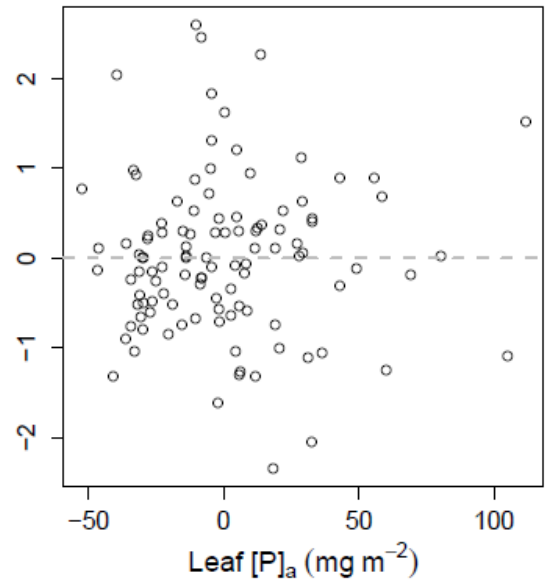


Table S4: Output of a comparative linear mixed effects model (after Eq. 2) with leaf traits expressed on a mass basis: fixed effects (top) and random effects (bottom). The top section shows fixed effect parameter estimates and associated standard error, degrees of freedom, test statistic and associated $p$-value. The final 'optimal' model is compared against a simpler 'null' model that includes only vegetation class ( $\mathbb{L}$ ) in the fixed component, but has an identical random term of Species nested within Site. Continuous explanatory variables were centred on their respective means.

\begin{tabular}{|c|c|c|c|c|c|c|c|c|c|c|}
\hline \multirow[b]{2}{*}{ Fixed effect } & \multicolumn{5}{|c|}{ Final model } & \multicolumn{5}{|c|}{ Null model } \\
\hline & Estimate & S.E. & DF & $\mathrm{t}$ value & $p$ value & Estimate & S.E. & DF & t value & $p$ value \\
\hline Forest (with other variables at mean value) & 210.04 & 14.81 & 71 & 14.181 & $<0.0001$ & 262.78 & 55.87 & 74 & 4.703 & $<0.0001$ \\
\hline Savanna (Vegetation contrast) & 86.35 & 28.33 & 5 & 3.048 & 0.0285 & -30.96 & 90.58 & 5 & -0.342 & 0.7464 \\
\hline Leaf $[\mathrm{N}]_{\mathrm{m}}$ & 6.95 & 2.14 & 71 & 3.252 & 0.0018 & & & & & \\
\hline Leaf $[P]_{m}$ & 104.52 & 43.46 & 71 & 2.405 & 0.0188 & & & & & \\
\hline Leaf $[\mathrm{N}]_{\mathrm{m}}:$ Leaf $[\mathrm{P}]_{\mathrm{m}}$ & 5.95 & 2.71 & 71 & 2.198 & 0.0312 & & & & & \\
\hline Random effect & Variance & $\%$ of total & & & & Variance & $\%$ of total & & & \\
\hline Intercept variance: Among sites & 0 & $0.0 \%$ & & & & 10,328 & $38.8 \%$ & & & \\
\hline Intercept variance: Among species & 2,513 & $32.4 \%$ & & & & 11,645 & $43.8 \%$ & & & \\
\hline \multirow[t]{2}{*}{ Residual (within species, within sites) } & 5,251 & $67.6 \%$ & & & & 4,627 & $17.4 \%$ & & & \\
\hline & 7,763 & $100.0 \%$ & & & & 26,600 & $100.0 \%$ & & & \\
\hline AIC & \multicolumn{5}{|c|}{1,228} & \multicolumn{5}{|c|}{1,275} \\
\hline Likelihood ratio test & \multicolumn{5}{|c|}{-606} & \multicolumn{5}{|c|}{-632} \\
\hline
\end{tabular}


Figure S3. Kernel density plot showing smoothed probability distributions of leaf mass per unit area ( $\mathrm{g}$ $\mathrm{m}^{-2}$ ) for each plant functional type.

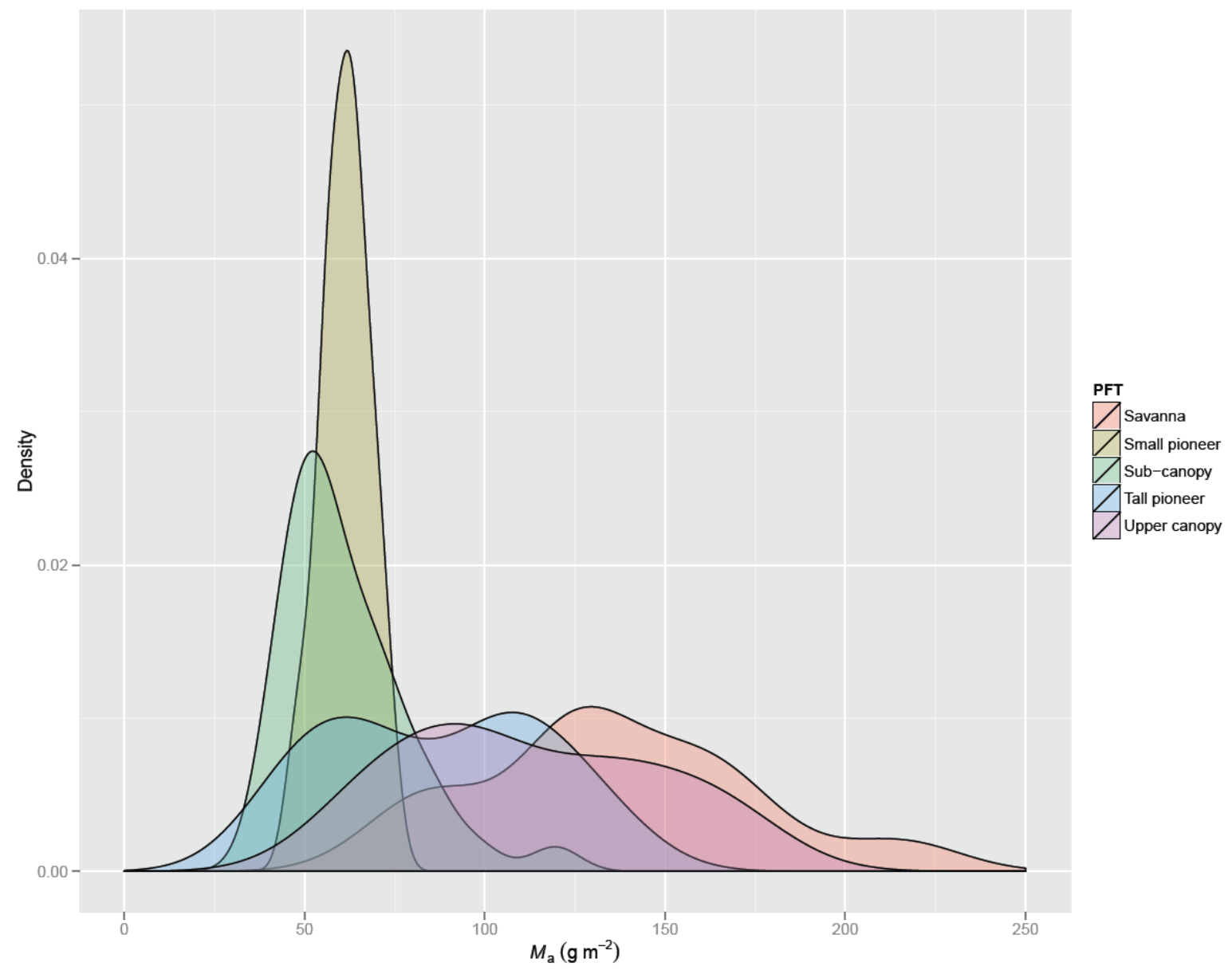

